\title{
Estrategias motivacionales para reforzar habilidades de emprendimiento
}

\section{Motivational strategies to reinforce entrepreneurial skills}

DOI: $10.46932 / \mathrm{sfjdv2n5-145}$

Received in: Oct 1st, 2021

Accepted in: Dec 30th, 2021

\section{Marjorie Magdalena Jara Jiménez}

Aspirante a Doctora en Educación en la Universidad Cesar Vallejo, Piura - Perú, Ingeniera en Gestión Empresarial. Magister en Administración de Empresas. Docente de Emprendimiento, Unidad Educativa Mocache, Mocache, Los Ríos, Ecuador.

Mocache km. 1 11/2 vía a Quevedo.

E-mail: clari_mar22@hotmail.com.

\section{Liliana Isabel Flores Anchundia}

Aspirante a Doctora en Educación en la Universidad Cesar Vallejo, Piura - Perú, Ingeniera en administración financiera. Magister en Contabilidad y Auditoría. Egresada en Maestría de Gestión Educativa. Docente en el Área de Contabilidad, Unidad Educativa José María Velasco Ibarra, Buena Fe, Los Ríos, Ecuador.

Escuela de Posgrado, programa académico doctorado en educación, Universidad Cercar Vallejo, Piura Perú.

calle arcadio fuente y transversal sin nombre junto al cementerio general.

E-mail: lili_isaldu@ hotmail.es.

\section{Victoria María Jiménez Ramos}

Licenciada en Ciencias de la Educación. Master en Ciencias de La Educación. Docente de la Unidad Educativa La Maná, Cotopaxi, Ecuador av. 19 de mayo y Jaime Roldos, La Maná.

E-mail: victoriamariajimenez@gmail.com

\section{Maira Magdalena Delgado Briones}

Ingeniera en Ecoturismo, Magíster en Educación con Mención en Pedagogía, docente del área de Emprendimiento y Gestión, Unidad Educativa 26 de Septiembre, Balzar, Guayas, Ecuador

Av. Del estudiante y Vinces, Balzar

E-mail: mairitadelgado@hotmail.es

\section{RESUMEN}

El presente trabajo de investigación se desarrolló teniendo como objetivo general proponer un manual de estrategias motivacionales para el fortalecimiento de las habilidades de emprendimiento de los estudiantes de bachillerato del Distrito 12D03 Mocache-Quevedo. La metodología se basó en un enfoque cuantitativo de tipo descriptivo proyectivo, con diseño de tipo no experimental, en donde se utilizó como técnica la entrevista, siendo el instrumento de recolección de datos el cuestionario, este estuvo dirigido a un grupo de estudio, integrado por 76 estudiantes, el mismo que estuvo constituido por 41 ítems, fue validado a través del juicio de expertos y la confiabilidad se realizó a través del coeficiente del Alfa de Cronbach, que arrojó un nivel de fiabilidad de 0,842. Los resultados permitieron conocer que los aspectos que debe contener un manual para reforzar las habilidades de emprendimiento deben estar relacionados con el auto concepto personal, auto estima y valoración a su capacidad, empatía ante situaciones adversas de sus pares 
y de ellos mismos, práctica de relaciones interpersonales, comunicación con sus pares, control de emociones ante discusiones y peleas entre compañeros, importancia del emprendimiento como alternativa laboral. En conclusión, se evidenció que es necesario el empleo de un manual de técnicas que permita el desarrollo de estrategias motivacionales para el fortalecimiento de las habilidades de emprendimiento de los estudiantes.

Palabras claves: Estrategias motivacionales, habilidades personales, habilidades sociales, habilidades de dirección, habilidades de emprendimiento.

\begin{abstract}
The present research work was developed with the general objective of proposing a manual of motivational strategies to strengthen the entrepreneurial skills of high school students from District 12D03 Mocache-Quevedo. The methodology was based on a projective descriptive quantitative approach, with a non-experimental design, where the interview was used as a technique, the questionnaire being the data collection instrument, this was aimed at a study group, composed of 76 students, which consisted of 41 items, was validated through the judgment of experts and the reliability was made through the Cronbach's Alpha coefficient, which yielded a reliability level of 0.842 . The results allowed us to know that the aspects that a manual should contain to reinforce entrepreneurial skills must be related to personal selfconcept, self-esteem and appreciation of their ability, empathy in the face of adverse situations of their peers and themselves, practice of relationships interpersonal, communication with their peers, control of emotions before discussions and fights between colleagues, importance of entrepreneurship as a work alternative. In conclusion, it was evidenced that it is necessary to use a manual of techniques that allows the development of motivational strategies to strengthen the entrepreneurial skills of students.
\end{abstract}

Keywords: Motivational strategies, personal skills, social skills, management skills, entrepreneurship skills.

\title{
1 INTRODUCCION
}

La condición de vida actual a raíz de la pandemia ha demostrado lo importante que representa el desarrollo de las habilidades de emprendimiento en los estudiantes, que le permiten la vinculación de los saberes obtenidos en el proceso educativos, además de la oportunidad de generar ingresos y mejorar su condición de vida y la de sus familiares.

Dentro del contexto educativo se orienta al estudiante en el área de emprendimiento más por diferentes factores no se estimula el desarrollo de las habilidades que le permitirán abrirse paso en esta actividad de una manera activa y próspera perdiendo el miedo a la situación y convirtiéndose en un ente futurista en pro del progreso y desarrollo, el docente orienta el quehacer educativo en base a la planificación que se encuentra estructurada en el material de lectura que ofrece el Gobierno nacional, pero es importante que este plantee estrategias motivacionales que favorezcan el desarrollo de las habilidades de emprendimiento de los estudiantes.

La base del problema radica en que el entorno que rodea al estudiante es hostil y por temor a enfrentar realidades como la crítica destructiva, la burla, y comentarios de alguien tipo despectivo u otras 
situaciones que no sólo desmoraliza también pueden resultar perturbadoras y frustrantes para el estudiante en formación y para el desempeño correcto de su emprendimiento; la no estimulación de las habilidades de emprendimiento como las habilidades sociales, habilidades de desarrollo, habilidades de competencias en el estudiantes generan dudas, miedo, inquietud, desinterés desgano y estos factores contribuyen a que el estudiante no tome la decisión de emprender, desvalorizando su autoestima elevando su temor a no cumplir con las expectativas de la sociedad insensible que le rodea.

A través de lo expuesto es importante destacar que, las instituciones educativas cuentan con la participación activa de los docentes, pero el temor de la participación de los estudiantes en el aula de clase, evidencia la falta de fortalecimiento de habilidades de emprendimiento, por este motivo el desarrollo de la investigación formula el siguiente problema ¿Qué estrategias motivacionales debe proponer un manual para el fortalecimiento de las habilidades de emprendimiento de los estudiantes de bachillerato? ¿Qué aspectos debe contener un manual para el fortalecimiento de las habilidades de emprendimiento de los estudiantes de bachillerato?

Tomando en consideración lo expuesto se plantea como objetivo general es proponer un manual de estrategias motivacionales para el fortalecimiento de las habilidades de emprendimiento de los estudiantes de bachillerato del Distrito 12D03 Mocache-Quevedo, 2021 y como objetivo específico, identificar los aspectos debe contener un manual para el fortalecimiento de las habilidades de emprendimiento de los estudiantes de bachillerato del Distrito 12D03 Mocache-Quevedo, 2021.

A continuación, se explican las teorías que sustentas la variable habilidades de emprendimiento, entre ellas tenemos la teoría del capital humano de Schultz (1961, como se citó en Pérez-Fuentes \& Castillo-Loaiza, 2016) en la que se sostiene que la formación del conocimiento y la experiencia, las cuales representan un valor más alto en relación con el éxito económico y empresarial frente a otros, por lo tanto se enfoca en estudiar aspectos de acuerdo con las capacidades y habilidades de las personas que se han adquirido durante su formación.

Por consiguiente, Sandoval \& Hernández (2018) señalan que la formación de conocimientos se encuentra en la actualidad, en la necesidad de fortalecer y desarrollar en los estudiantes; competencias que garanticen el correcto fortalecimiento de las destrezas y habilidades centradas en los estudiantes para que se relacionen y practiquen en la realidad. De tal manera que, para esta teoría, el capital humano está compuesto por el conocimiento adquirido y las habilidades obtenidas a través de la experiencia, teniendo en cuenta que para la satisfacción de los objetivos empresariales tanto el conocimiento como lo las capacidades deben ejercerse conjuntamente.

Las habilidades de emprendimiento se sustentan en la teoría de las tres necesidades de David McClelland (1960) quien sostuvo que todas las personas poseen tres necesidades básicas: Necesidad de logro: Se refiere al esfuerzo por sobresalir, el logro en relación con un grupo de estándares, la lucha por 
el éxito. Necesidad de afiliación: Se refiere al deseo de relacionarse con las demás personas, es decir de entablar relaciones interpersonales amistosas y cercanas con los demás integrantes de la organización y Necesidad de poder/influencia: Se refiere a la necesidad de conseguir que las demás personas se comporten en una manera que no lo harían, es decir se refiere al deseo de tener impacto, de influir y controlar a los demás (Padovan 2020).

El manejo de las habilidades personales es fundamental para desarrollar el cuidado personal, gestionar y dirigir a los demás y lograr resultados satisfactorios. En este contexto, el emprendedor debe ser capaz de optimizar sus habilidades y facilitar su comportamiento mediante factores motivacionales, sentimientos adecuadamente constituidos y actitudes que le faciliten la identificación de situaciones con el mayor control y dominio de las emociones. (Ferro, 2019)

A partir de esta teoría surgen las dimensiones de las habilidades emprendedoras (que en cierta medida también corresponde a las de Maslow, de quien Mc Clelland es seguidor). Estas dimensiones son: habilidades personales, habilidades sociales y habilidades directivas. (Hernández, et al. 2016)

La primera según Loayza-Borda (2021) se refiere a la capacidad que posee cada persona para aprender, haciendo uso de sus habilidades y actitudes para emprender gestiones haciendo buen uso de los recursos y del tiempo. La segunda dimensión es habilidades sociales, que se acuerdo con Balcázar (2021) están vinculadas con la comunicación, la capacidad de negociar y establecer interrelaciones productivas, trabajar en equipo siento dinámicos, asertivos y justos ante la resolución de conflictos. Finalmente, la dimensión habilidades directivas cuya funcionalidad está enfocada en la motivación, en la capacidad de orientación y liderazgo. (Paredes-Pérez, et al., 2021)

La variable estrategias de motivación se sustenta en teorías que amplían las vías de comprensión de su impacto en el desarrollo de las habilidades de los estudiantes. La primera es la teoría de modificación de las conductas de Skinner (/0000) la cual explica que toda persona reacciona de acuerdo al estímulo que recibe del ambiente externo. (Lima \& Araújo, 2018)

Según Carrasco \& Prieto (2018) estos estímulos se los reconoce como refuerzo, qué pueden ser negativos o positivos, efectivamente la recompensa es otorgada ante un comportamiento productivo que ha logrado alcanzar las expectativas, mientras que el refuerzo negativo está vinculado con la reprensión ante la inadecuada conducta

La investigación principalmente se basa en la teoría sociocultural de Vygotsky (1981), que atribuye mayor importancia a la interacción social, como principal factor del aprendizaje cognoscitivo. Según esta teoría el aprendizaje en la etapa escolar debe ser autónomo, pero a la vez guiado a partir de herramientas que faciliten el trayecto por el camino del aprendizaje. (Santana, et al., 2021)

Según Solveira et al (2020), las estrategias motivacionales son procedimientos didácticos y dinámicos que se aplican en el proceso de enseñanzas a través de recursos y técnicas específicas, que 
promueven la mejora del estado de ánimo de los estudiantes, lográndose un estímulo en el cumplimiento de sus deberes y obligaciones en el aula de clase. Aquí es donde el docente con sus experiencias señala la importancia de la asignatura y de los beneficios que conlleva participar en cada uno de los procesos de formación.

En su escrito Valenzuela et al (2018) conceptualizan a la motivación como factor determinante para el desarrollo psicológico correcto del estudiante en los procesos de aprendizaje y también de mucha importancia en el ámbito educativo, por ello es elemental que se apliquen estrategias motivacionales encaminadas a mejorar las acciones, actitudes y su percepción (p. 9).

En este aspecto resulta imprescindible en la actualidad que los docentes empleen estrategias motivacionales que brinden garantías de estabilidad emocional, salud mental y física, y aún más sientan agrado por estudiar para aprender para la vida. A continuación, se explican las estrategias que favorecerán el nivel de motivación en el aprendizaje:

Gamificación: para Pertegal \& Lorenzo (2019) es una herramienta metodológica o técnica de aprendizaje diseñada en para mejorar los procesos de formación académico, por medio de un conjunto de recurso creados precisamente para ayudar a mejorar el rendimiento escolar en los estudiantes (p. 554).

Estrategias vivenciales: para Lara (2018) las vivenciales son todas aquellas experiencias, trabajo, conocimiento, comportamientos, acciones, valores, principio y aprendizajes anteriores, que se aplican en cualquier proceso de formación, en una conversación, en la ejecución de proyectos y en los trabajos (p. 48).

Según, Torres, et al. (2017) optar por técnicas que estimulen o ayuden a superar problemas de malos aprendizajes, las estrategias vivenciales son el medio por el cual el docente brinda orientación motivacional que dan esperanza a los estudiantes a aprender motivados.

Estrategias afectivas: las estrategias afectivas, como hablarse así mismo, hacerse una autovaloración de sí mismo positivamente, visualizar oportunidades para emplear métodos correctos y cumplirlos y un conjunto de acciones para mejorar sus emociones y las actividades de aprendizajes, tienen como objetivos la motivación intrínseca que aumente lo efectos relacionados con el mejoramiento de los aprendizajes.

Según Ayala (2019) las estrategias afectivas son acciones que el estudiante realiza para mejorar y comprender mejor los procesos de aprendizajes, es un conjunto de estímulos psicológicos que propician el crecimiento personal, social y formativo.

Por otra parte, Morales \& Curiel (2019) las estrategias afectivas son ideales para la reflexión personal, concientización y el crecimiento de valores sociales, personales y comunicativos dentro y fuera del aula de clase de los estudiantes, por ente la institución debe emplear estrategias de motivación afectivas para los estudiantes y para el confort de sus aprendizajes. 


\section{METODOLOGIA}

La presente investigación fue descriptiva, con un enfoque cuantitativo, de tipo no experimental con un diseño proyectivo aplicado, para Guevara, Verdesoto, \& Castro Nelly (2020) esta investigación tiene la finalidad de relatar rasgos esenciales de fenómenos de estudio, usando características fundamentales de conjuntos homogéneos de fenómenos, utilizando razonamientos que permitan la estructura o conducta del dicho fenómeno. Por otra parte, Cadena (2017), señala que el enfoque cuantitativo es aquel donde se recogen datos para realizar el análisis respecto al objeto de estudio, por otro lado, Rodríguez (2017), manifiesta que las investigaciones cuantitativas estudian acontecimientos observables.

Por lo tanto, en esta investigación se recogió datos, que permitieron conocer las características del objeto de estudio para aplicar las estrategias motivacionales adecuadas que fortalecerán las habilidades de emprendimiento de los estudiantes, y al mismo tiempo mejorará la educación de los estudiantes.

Fue de diseño proyectivo o propositivo por cuanto se permitió la manipulación de al menos una variable (Pastrano. 2019) en este caso la variable fática y el análisis que la misma posee en la variable teórica solamente permite la diferencia de los experimentos en el grado de seguridad o confiabilidad que pueda tomarse en cuenta sobre las equivalencias iniciales de los grupos. Para el desarrollo de este diseño metodológico se aplicó la prueba piloto para el nivel de confiabilidad y el diagnóstico con las encuestas dirigidas a los estudiantes.

\section{RESULTADOS}

OE: Identificar los aspectos debe contener un manual para el fortalecimiento de las habilidades de emprendimiento de los estudiantes de bachillerato.

Tabla 01: Aspectos debe contener un manual para el fortalecimiento de las habilidades de emprendimiento.

\begin{tabular}{|c|c|c|}
\hline DIMENSIÓN & TABLA & ELEMENTO \\
\hline \multirow{4}{*}{$\begin{array}{l}\text { Habilidades } \\
\text { personales }\end{array}$} & & Bajo nivel de auto concepto personal. \\
\hline & $\mathrm{N}^{\circ} 4$ & Bajo nivel de auto estima y poca valoración a su capacidad \\
\hline & & El temor a enfrentarse a resolver problemas. \\
\hline & $\mathrm{N}^{\circ} 6$ & $\begin{array}{l}\text { Falta de empatía ante situaciones adversas de sus pares y de } \\
\text { ellos mismos }\end{array}$ \\
\hline \multirow[t]{2}{*}{ Habilidades sociales } & \multirow[t]{2}{*}{$\mathrm{N}^{\circ} 7$} & Escasa práctica de relaciones interpersonales. \\
\hline & & Escaso nivel de comunicación con sus pares \\
\hline $\begin{array}{l}\text { Habilidades } \\
\text { Dirección }\end{array}$ & $\mathrm{N}^{\circ} 10$ & $\begin{array}{l}\text { Escaso control de emociones ante discusiones y peleas entre } \\
\text { compañeros. } \\
\text { Desconocimiento de la importancia del emprendimiento como } \\
\text { alternativa laboral. }\end{array}$ \\
\hline
\end{tabular}


Los datos que reflejan la tabla 01 muestran los aspectos que debe contener un manual para el fortalecimiento de habilidades de emprendimiento de los estudiantes de Bachillerato entre ellos, el auto concepto personal, auto estima y valoración a su capacidad, empatía ante situaciones adversas de sus pares y de ellos mismos, práctica de relaciones interpersonales, comunicación con sus pares, control de emociones ante discusiones y peleas entre compañeros, importancia del emprendimiento como alternativa laboral.

OG: proponer un manual de estrategias motivacionales para el fortalecimiento de las habilidades de emprendimiento de los estudiantes de bachillerato del Distrito 12D03 Mocache-Quevedo.

\section{MANUAL DE ESTRATEGIAS MOTIVACIONALES PARA FORTALECER LAS HABILIDADES DE EMPRENDIMIENTO EN ESTUDIANTES DE BACHILLERATO DEL DISTRITO 12D03 QUEVEDO - MOCACHE.}

\section{DATOS GENERALES:}

1.1. Institución: Distrito 12D03 Quevedo - Mocache.

1.2. Denominación: Manual de estrategias motivacionales

1.3. Población (Cobertura): Estudiantes de Bachillerato

1.4. Responsable: MSc. Jara Jiménez, Marjorie Magdalena

1.5. Duración: Dos (02) semanas.

\section{JUSTIFICACIÓN}

El presente manual se fundamenta en el concepto que McClelland (1960) esbozo en la llamada teoría de las tres necesidades en la que sostenía que todas las personas poseemos tres necesidades básicas: necesidad de logro, necesidad de afiliación y necesidad de poder/influencia (Padovan 2020).

Consideramos que a través de las actividades aquí desarrolladas los estudiantes se puedan motivar a pensar, reflexionar sobre el emprendimiento futuro personal o colectivo. Consideramos que los temas tratados en estas ocho (08) sesiones permitirán a los estudiantes no solo conocerse a nivel personal, sino también a conocer que todo lo pueden hacer si reconocen quienes son, cuáles son sus fortalezas, sus debilidades y sus oportunidades de proyectar un negocio, una empresa u otro proyecto emprendedor que beneficie su vida.

Finalmente, el manual se propone desarrollar iniciativas tendientes a apoyar e incentivar y mejorar las habilidades emprendedoras de los estudiantes de bachillerato del Distrito 12D03 Quevedo - Mocache. 


\section{FUNDAMENTACIÓN}

\subsection{FUNDAMENTO TEÓRICO}

La escuela entendida como un espacio de transmisión de conocimiento y formación de hábitos, habilidades, capacidades y competencias, tiene en el emprendimiento uno de los tópicos poco desarrollados

Se parte del concepto de emprender como llevar a cabo una actividad que supone cierto reto y riesgo y que implica tomar decisiones y llevarlas a cabo. Cada vez que alguien asume un emprendimiento está favoreciendo dos aspectos individuales y personales: la auto realización: (el sentir que haces algo que vale la pena, que te llena, la elección o toma de decisiones, hacer lo que te gusta) y la autonomía económica (ganar suficiente dinero para vivir como te gustaría).

Sobre la base de estos conceptos el presente manual se diseña como una forma de mejorar las capacidades y habilidades emprendedoras de los estudiantes de bachillerato, a fin de generar en ellos, una motivación desde la óptica de las llamadas estrategias motivacionales, entendidas como procedimientos que utilizan los estudiantes durante su proceso de aprendizaje para incidir y gestionar su propia motivación y afectividad. Estas estrategias en sí, no están directamente relacionadas con los contenidos de clase cotidianos, en realidad son el soporte pata que los estudiantes puedan optimizar sus aprendizajes en el aula, incorporando nuevas formas de aprender y de trabajar con contenidos más asociados a la parte afectiva como lo son las habilidades emprendedoras.

\subsection{FUNDAMENTO FILOSÓFICO}

La vida en si requiere que todos vivamos en compañía de uno y otros. La convivencia requiere que las personas tengamos ciertas habilidades para poder interrelacionarnos con nuestros pares, en momentos de alegría, tristeza, o discusión. Poseer esas habilidades requiere aprendizaje, aprender a convivir con los demás es una necesidad que, desde la escuela, debemos desarrollar en los estudiantes, pues su vida posterior fuera de ella estará llena de avatares y desafíos, en los que, si no poseen habilidades sociales positivas, difícilmente podrán ser entendidos, escuchados. Por eso el presente manual apunta a desarrollar estas habilidades en los estudiantes, a fin de que puedan ponerlas en práctica durante cualquier emprendimiento que realicen en su vida después de abandonar la escuela, y les sirva para su desarrollo óptimo como personas.

\subsection{ENFOQUES METODOLÓGICOS}

En lo metodológico el presente manual e s un aporte a la didáctica de la enseñanza docente y al aprendizaje de habilidades sociales de los estudiantes., pues creemos que en este contexto a actual 
pandémico, urge renovar el conocimiento y practica de habilidades sociales desvalorizadas por el enclaustramiento obligado de nuestros jóvenes.

Por otro lado, la importancia de la aplicación del manual de habilidades sociales radica en analizar algunos elementos extraños que sirvan para impulsar o limitar el entrenamiento en habilidades sociales conociendo así algunos criterios que sirvan para optimizar la efectividad del manual en mención, considerando además que toda investigación se convierte en información que puede ser utilizada en otros estudios con las mismas variables de estudio

\subsection{FUNDAMENTO PSICOPEDAGÓGICO}

El ámbito escolar es el medio donde además de promover el aprendizaje y el desarrollo de actitudes y aptitudes adecuadas para el de desarrollo de los estudiantes, es también el espacio donde se les enseña a convivir y vivir en armonía con sus pares

Acorde con lo destacado en 1998 en el informe Delors, todo sistema educativo debe promover entre los estudiantes aprendizajes para la vida sustentados en los cuatro pilares que deben sustentar toda educación como son: aprender a aprender; aprender a hacer; aprender a ser y aprender a vivir juntos; en la manifiesta que es imprescindible incluir además de los aspectos cognoscitivos e intelectuales, aspectos relacionados con el desarrollo personal y con las habilidades sociales resaltando la importancia que tiene para la educación el aprender a vivir juntos, es decir a "convivir” (Yanac,2019).

\subsection{FUNDAMENTO LEGAL}

El fundamento legal del presente manual se sustenta en lo establecido en la constitución política del Ecuador, que en el artículo 66 refiriéndose a la educación establecer que "La educación, inspirada en principios éticos, pluralistas, democráticos, humanistas y científicos, promoverá el respeto a los derechos humanos, desarrollará un pensamiento crítico, fomentará el civismo; proporcionará destrezas para la eficiencia en el trabajo y la producción; estimulará la creatividad y el pleno desarrollo de la personalidad y las especiales habilidades de cada persona; impulsará la interculturalidad, la solidaridad y la paz."

Asimismo, se fundamenta en los aspectos metodológicos y curriculares inspirados en la Ley General de Educación No 127

\section{OBJETIVO}

Motivar a los estudiantes de bachillerato para que identifiquen sus necesidades personales, sociales y de logro en relación a las habilidades emprendedoras sus capacidades y habilidades emprendedoras y así procurar asuman una cultura de emprendedora que mejore su calidad y condición de vida. 


\section{ANÁLISIS DE FACTIBILIDAD DE LA EJECUCIÓN:}

El manual tiene un 100\% de aplicabilidad en su ejecución, pues la coordinación con la dirección de la institución es directa, además, la problemática detectada es de conocimiento de todos los directivos del distrito educativo, y en ese sentido, se tiene la necesidad de aportar a la solución del problema, con documentos innovadores como el presente manual. Por otra parte, el desarrollo de las actividades no demanda mucho gasto económico, pero sí de tiempo, porque el manual con sus actividades se debería aplicarse en paralelo a las clases normales.

\section{METODOLOGÍA (Método que se sugiere utilizar)}

El manual de estrategias motivacionales está diseñado con el fin de apoyar, incentivar y mejorar las capacidades y habilidades emprendedoras fortalecer las habilidades y capacidades emprendedoras de los estudiantes de bachillerato del Distrito 12D03 Quevedo - Mocache. La metodología del programa se caracteriza por:

El manual procederá aplicar un instrumento diagnostico (Cuestionario) antes (pre test) y después (post test) del programa, a fin de evidenciar los cambios producidos en los aprendizajes y el desarrollo de sus habilidades emprendedoras.

El manual se desarrollará mediante sesiones de enseñanza aprendizaje con una duración 1 hora pedagógica (45min).

El manual está constituido por ocho (08) sesiones individuales, para dar pie, después, a charlas informativas por grupos. Con ello se buscará, buscando la homogeneidad para explicar a cada grupo las pautas educacionales adaptadas a la situación.

La frecuencia de las sesiones será realizada de acuerdo con las coordinaciones con la dirección de la institución educativa sobre su calendarización.

Se usará un lenguaje de fácil comprensión para el estudiantado y se diseñaran situaciones de aprendizaje en que este puede participar activamente.

Se aplicarán diversas metodologías de enseñanza, caracterizándose por la aplicación de los métodos siguientes: Dinámica grupal, Role playing, Recursos Multimedia, Debate, Lecturas. Seleccionadas, Ficha de auto evaluación., Hojas de trabajo, etc.

Finalmente se procederá a compilar la información y redactar el informe final de la investigación.

La aplicación del manual considera los principios éticos en la interacción con los colaboradores en la investigación para lo cual se solicitará el permiso explicando detalladamente el trabajo de investigación a realizarse y asegurando el compromiso de confidencialidad 
10 ACTIVIDADES

\begin{tabular}{|c|c|c|c|c|c|c|c|c|}
\hline Tiempo & & & & & & & & \\
\hline es Actividad & 1 & 2 & 3 & 4 & 5 & 6 & 7 & 8 \\
\hline \begin{tabular}{lr}
\multicolumn{1}{c}{1.} & \\
Coordinación & con \\
directivos & $y$ \\
estudiantes &
\end{tabular} & $\mathbf{X}$ & & & & & & & \\
\hline $\begin{array}{rr}\text { Sesión } & 1: \\
\text { Conociéndonos } & \end{array}$ & & $\mathbf{X}$ & & & & & & \\
\hline $\begin{array}{c}\text { Sesión 2: } \\
\text { Nuestra autoestima }\end{array}$ & & & $\mathbf{X}$ & & & & & \\
\hline $\begin{array}{l}\quad \text { Sesión 3: } \\
\text { Enfrentemos } \\
\text { nuestros temores y } \\
\text { problemas }\end{array}$ & & & $\mathbf{X}$ & & & & & \\
\hline $\begin{array}{c}\text { Sesión 4: } \\
\text { Nuestras emociones }\end{array}$ & & & & $\mathbf{X}$ & & & & \\
\hline \begin{tabular}{l}
\multicolumn{1}{c}{ Sesión $5:$} \\
Solucionando \\
problemas \\
interpersonales
\end{tabular} & & & & $\mathbf{X}$ & & & & \\
\hline \begin{tabular}{lr}
\multicolumn{1}{c}{ Sesión } & 6: \\
Aprendiendo & a \\
conversar en grupo
\end{tabular} & & & & & $\mathbf{X}$ & & & \\
\hline \begin{tabular}{lr}
\multicolumn{1}{c}{ Sesión } & $7:$ \\
Seamos empáticos \\
aprendiendo & a \\
cooperar & y \\
compartir &
\end{tabular} & & & & & $\mathbf{X}$ & & & \\
\hline \begin{tabular}{l}
\multicolumn{1}{c}{ Sesión 8} \\
Mi emprendimiento \\
laboral
\end{tabular} & & & & & & $\mathbf{X}$ & & \\
\hline \begin{tabular}{l}
\multicolumn{1}{c}{10.} \\
Aplicación del pre \\
test a fin de evaluar \\
eficacia del manual \\
aplicado
\end{tabular} & & & & & & & $\mathbf{X}$ & \\
\hline \begin{tabular}{l}
\multicolumn{1}{c}{11.} \\
Elaboración \\
informe
\end{tabular} & & & & & & & & $\bar{X}$ \\
\hline
\end{tabular}

\section{DISCUSION}

Entre las características de la toma de decisiones, según la dimensión de las habilidades de dirección. En los resultados se evidenció que existe un grupo de estudiantes no asumen sus propias conclusiones ni tienen capacidad para decidir rápidamente y cuando toman decisiones lo hacen sin considerar la opinión de los demás ni asumir riesgos. En relación a las habilidades de dirección, se entiende que estas son aquellas que aumentan la productividad de los estudiantes, sino que también da un buen ejemplo en el desarrollo de conocimientos. A través de ello se pueden detectar las fortalezas de los estudiantes y alentarlos a desarrollar sus habilidades. Según lo analizado en la investigación se pudo conocer que un grupo de estudiantes no son justos, optimistas, no tienen carisma ni autocontrol de sus 
emociones por tanto no tienen capacidad para planificar y llevar una buena organización de sus actividades.

Estos resultados se relacionan con los encontrados en el estudio de Herrera, (2020) llevó a cabo el estudio sobre "El rendimiento y la motivación para el emprendimiento de los estudiantes en el bachillerato técnico". En el cual se indica que los docentes tienen habilidades necesarias para fortalecer la motivación para el emprendimiento de los estudiantes. Se reflejaron diferencias en el aprendizaje, presentándose un incremento del esfuerzo para actividades relacionadas con las tutorías en línea, uso de herramientas de colaboración y comunicación para el emprendimiento. Por lo tanto, se expresa que existe significancia entre las habilidades de dirección como la toma de decisiones y el liderazgo.

Por otra parte, se encuentran las características del liderazgo, según la dimensión de las habilidades de dirección. Los resultados reflejaron que un grupo de estudiantes no son justos, optimistas, no tienen carisma ni autocontrol de sus emociones por tanto no tienen capacidad para planificar y llevar una buena organización de sus actividades. Con referencia a las habilidades de dirección y el liderazgo se pudo analizar que los líderes dentro de las escuelas deben trabajar para comprender las necesidades y habilidades de desarrollo personal de los estudiantes, así como las brechas de conocimiento. Esto puede ayudar a crear una cultura de aprendizaje y responsabilidad, predicando con el ejemplo para demostrar cómo el estudiante puede mejorar su capacidad.

Este estudio se relaciona con Valenzuela, Muñoz, \& Montoya (2018) quienes investigaron sobre las "estrategias motivacionales efectivas en profesores en formación" cuyas dimensiones de análisis fueron la motivación hacia el aprendizaje, motivación con la relación de tareas y motivación como entretención. Se concluyó que, los líderes en las escuelas deben asegurarse de que una cultura de investigación, innovación y creatividad esté en el corazón de la organización, y deben buscar comentarios y datos regulares sobre el desempeño de los estudiantes en relación con los cambios.

Finalmente, los datos que reflejan la tabla 01 muestran los aspectos que debe contener un manual para el fortalecimiento de habilidades de emprendimiento de los estudiantes de Bachillerato entre ellos, el auto concepto personal, auto estima y valoración a su capacidad, empatía ante situaciones adversas de sus pares y de ellos mismos, práctica de relaciones interpersonales, comunicación con sus pares, control de emociones ante discusiones y peleas entre compañeros, importancia del emprendimiento como alternativa laboral.

Los resultados que se obtuvieron fueron basados en una investigación de tipo descriptivo, de tipo no experimental, con diseño proyectivo, donde se propuso un manual de estrategia motivacionales para fortalecer habilidades de emprendimiento en estudiantes de bachillerato, sin embargo queda la interrogante ¿La aplicación del manual de estrategias motivacionales para estudiantes de bachillerato 
fortalecerá las habilidades personales, sociales y de dirección de los estudiantes?, la misma que daría paso a una nueva investigación.

\section{CONCLUSIONES}

En conclusión, es importante considerar el desarrollo de las habilidades personales como la iniciativa, confianza, motivación y comunicación que generan emprendimientos en los estudiantes de bachillerato. Es de suma importancia desarrollar la comunicación porque tiene un impacto significativo en el bienestar y capacidad para tener éxito académico.

$\mathrm{Al}$ examinar las habilidades sociales mediante las relaciones personales y trabajo en equipo se fortalecen las habilidades de los estudiantes de bachillerato. En este caso, trabajar en equipo en diferentes asignaciones minimiza las cargas de trabajo para todos los estudiantes, al compartir ideas y responsabilidades, lo que permite que sean críticos al momento de desarrollar las tareas designadas.

Al analizar las habilidades de dirección como la toma de decisiones y el liderazgo se podrá fortalecer el emprendimiento de los estudiantes de bachillerato. Se aplica porque la decisión de colaboración permite a los estudiantes interactuar entre sí en un proyecto. Esta interacción es una gran oportunidad de aprendizaje para adquirir habilidades que no tenían y volverse más experimentados en el emprendimiento.

Por último, se concluye que, un manual para el fortalecimiento de las habilidades de emprendimiento de los estudiantes de bachillerato debe contener actividades que fortalezcan las habilidades personales, sociales y de dirección en los estudiantes de forma que se logre superar dificultades de aprendizajes. 


\section{REFERENCIAS}

Ayala, E. (2019). Experiential participatory music education strategy in the quality of training for early childhood students. Revista Iberoamericana para la inevstigación y el desarrollo Educativo, 10(19), 118. doi:https://doi.org/10.23913/ride.v10i19.576

Balcázar, A. (2021). The educational camp as a setting for strengthening social skills. Retos(41), 143-153.

Cadena, P., Rendón, R., Aguilar, J., Salinas, E., Cruz, F., \& Sangerman, D. (2017). Métodos cuantitativos, métodos cualitativos o su combinación en la investigación: un acercamiento en las ciencias sociales. Revista mexicana de ciencias agrícolas, 8(7), 1603-1617. México.

Carrasco, M., \& Prieto, M. (2018). Skinner, contribuciones del conductismo a la educación. Grandes de la educación (387), 77-79. doi:pym.i367.y2016.014

Ferro, J. (2019). Trabajo en equipo para mejorar la calidad laboral. Universidad Católica de Colombia. Bogotá : Universidad Católica de Colombia. Obtenido de https://repository.ucatolica.edu.co/bitstream/10983/24011/1/TRABAJO\%20EN\%20EQUIPO\%20PARA \%20MEJORAR\%20LA\%20CALIDAD\%20LABORAL.pdf

Guevara, G., Verdesoto, A., \& Castro Nelly. (2020). Metodologías de investigación educativa (descriptivas, experimentales, participativas, y de investigación-acción). RECIMUNDO, 164.

Hernàndez, J., Espinoza, J., \& Aguilar, M. (2016). Differences in motivators and values in the work of maquiladora industry employees. 61(1), 58-83. doi:https://doi.org/10.1016/j.cya.2015.09.003

Herrera, M. (2020). El rendimiento y la motivación de los estudiantes en el bachillerato técnico. Interciencia, v.31 n.3 .

Lara, F. (2018). Experiential strategies to promote values in first grade students in the area of religious education of the educational institution José Contreras Cabrera de Pomacucho. UNHEVAL, 45-68. doi:oai:172.16.0.151:UNHEVAL/2924

Lima, F., \& Araújo, E. (2018). Condicionamento operante de Skinner: a influência do desempe-nho no estágio de operações na selva da siesp. Revista Agulhas Negras, 2(2), 62-70. Obtenido de http://ebrevistas.eb.mil.br/aman/article/view/1874/1513

Loayza-Borda, J. (2021). Critical thinking programs as a soft skill in education. Maestro y Sociedad, 18(3), 1137-1148.

Obtenido

de

https://maestroysociedad.uo.edu.cu/index.php/MyS/article/view/5399/5056

Morales, R., \& Curiel, L. (2019). Socio-affective strategies feasible to apply in virtual learning environments . Edutec, Revista Electrónica de Tecnología Educativa, 36-52. doi: https://doi.org/10.21556/edutec.2019.69.1289

Paredes-Pérez, M., Cárdenas-Tapia, V., \& Palomino-Crispin, A. (2021). Habilidades directivas y planificación estratégica en una universidad peruana. Gaceta Científica, 7(1), 17-22. Obtenido de https://doi.org/10.46794/gacien.7.1.1061

Pérez-Fuentes, D., \& Castillo-Loaiza, J. (2016). Human capital, theories and methods: importance of the health variable. Econ. soc. territ, 16(52), 651-673. Obtenido de 
http://www.scielo.org.mx/scielo.php?script=sci_arttext\&pid=S1405-

$84212016000300651 \& \operatorname{lng}=$ es\&tlng=es.

Pertegal, L., \& Lorenzo, G. (2019). Gamification in the classroom through the TIC. INFAD, 553-562. doi:10.17060/ijodaep.2019.n1.v3.1535

Rodriguez, M. (2017). Lineamientos teóricos y metodológicos de la investigación cuantitativa en ciencias sociales. In Crescendo, 8(1), 115-121. Obtenido de file:///C:/Users/PERSONAL/Downloads/DialnetLineamientosTeoricos YMetodologicosDeLaInvestigacio-6042332.pdf

Sandoval, J., \& Hernández, G. (2018). Crítica a la teoría del capital humano, educación y desarrollo socioeconómico. Revista Ensayos Pedagógigos, 1-20. doi:http://dx.doi.org/10.15359/rep.13-2.7

Santana, R., Izaguirre, R., \& Reyes, J. (2021). Influence of the sociocultural context in the development of historical thought. Opuntia Brava, 13(1), 232-245. Obtenido de http://200.14.53.83/index.php/opuntiabrava/article/view/1382

Solveira, F., Cambre, M., Roland, G., \& Baldriz, I. (6 de 11 de 2020). Estrategias de motivación con adolescentes en Espacio Ciencia (Uruguay). INNOTEC GESTIÓN, 1-10. doi:https://doi.org/10.22323/3.03020202

Torres, D., Fonseca, W., \& Pineda, B. (2017). Experiences as a Strategy for Strengthen Critical $\square$ inking in. Praxis \& Saber, 8(17), 201 - 220. doi:https://doi.org/10.19053/22160159.v8.n17.2018.7207

Valenzuela, J., Muñoz, C., \& Montoya, A. (2018). Effective motivational strategies in pre-service teachers. Educação e Pesquisa, 1-20. doi:https://doi.org/10.1590/S1678-4634201844179652 